\title{
Subwavelength vacuum lattices and atom-atom interactions in two-dimensional photonic crystals
}

\author{
A. González-Tudela1, C.-L. Hung ${ }^{2,3}$, D. E. Chang4, J. I. Cirac ${ }^{1}$ and H. J. Kimble
}

\begin{abstract}
Quantum simulation with cold atoms in optical lattices is an attractive avenue for explorations of quantum many-body physics. A principal challenge in the field is to increase the energy and length scales in current set-ups, thereby reducing temperature and coherence-time requirements. Here, we present a new paradigm for high-density, two-dimensional optical lattices in photonic crystal waveguides. Specially engineered two-dimensional photonic crystals provide a practical platform to trap atoms and engineer their interactions in ways that surpass the limitations of current technologies and enable investigations of novel quantum many-body matter. Our schemes remove the constraint on the lattice constant set by the free-space optical wavelength in favour of deeply sub-wavelength atomic arrays. We further describe possibilities for atom-atom interactions mediated by photons in two-dimensional photonic crystal waveguides with energy scales several orders of magnitude larger than for exchange interactions in free-space lattices and with the capability to engineer strongly long-range interactions.
\end{abstract}

( ) bstacles to the exploration of quantum many-body physics with cold atoms ${ }^{1,2}$ include the small energy scales $\left(\sim 10^{3}-10^{4} \mathrm{~Hz}\right)$ and the restriction to nearest-neighbour interactions in free-space optical lattices. Alternative approaches for quantum simulation include dipolar molecules ${ }^{3}$ and Rydberg atoms $s^{4,5}$, which give rise to weakly long-range interactions that typically scale as $1 / r^{\alpha}$, where $\alpha=3$ and $r$ is the distance between the atoms (in standard notation ${ }^{6}$, weakly (strongly) long-range interactions are for $\alpha>D(\alpha<D)$ with $D$ the dimensionality). Other possibilities involve lattices with a period below that of a free-space optical standing wave by using plasmonic $^{7}$ or superconducting systems ${ }^{8}$.

In this Article we show that the integration of ultra-cold atomic physics with nanophotonics opens up new avenues for the creation of quantum many-body matter. As illustrated in Fig. 1a, two-dimensional arrays of atom traps can be generated by optical-dipole forces from guided modes (GMs) of photonic crystal waveguides (PCWs) whose refractive index $n(\mathbf{r})$ is modulated with a period $d<\lambda_{0} / 2$, where $\lambda_{0}$ is the vacuum wavelength ${ }^{9}$. Atoms can also be trapped in two-dimensional vacuum lattices arising from the spatial variation of Casimir-Polder (CP) forces ${ }^{10}$ near a PCW with lattice constant $d \approx 50 \mathrm{~nm}$. Such atom lattices with $d<\lambda_{0} / 2$ yield larger energy scales for quantum simulation than is generally possible with conventional free-space optical lattices.

We further show that PCWs provide a versatile means for creating atom-atom interactions mediated by photons within the GMs of the PCW. These effective atomic interactions can be very large and strongly long-range. By operating with a Raman transition either within a bandgap or in a dispersive regime for the PCW, the dynamics of atom-atom interactions can be predominantly conservative or dissipative, with the possibility to make this choice in real time. Finally, we analyse the prospects of this scheme to implement spin models for quantum simulation.

Our analyses with two-dimensional structures are based on early treatments of atom-photon interactions in general photonic crystal structures $^{11-14}$ and are motivated by recent experiments for atom localization near nanoscopic dielectrics ${ }^{15-20}$ and related theoretical proposals (for example, self-organization ${ }^{21}$ and coherent atom-atom interactions ${ }^{14,22}$ ). By advancing atomic lattices with PCWs from one to two dimensions, we gain access to a richer set of phenomena, including Hubbard physics with large interaction energies, quantum magnetism with the capability to design the strength and range of the interaction, and topological phases for two-dimensional atom lattices with photon-mediated interactions ${ }^{23-25}$.

Relevant to our work with two-dimensional structures is ref. 22 (for one-dimensional PCWs), which provides an initial elaboration of the principles for achieving long-range coupling between atoms in realistic structures ${ }^{18,19}$. Carefully crafted trapping schemes are key to enabling experimental realizations, and such schemes for two dimensions are presented here for the first time, including deeply subwavelength vacuum lattices. We extend the atomic-level schemes and mode-coupling strategies to achieve engineered longrange interactions and dynamic tunability for two-dimensional PCWs, and provide realistic analyses to quantify them. By extending the ideas introduced in ref. 22 we include loss mechanisms from free-space atomic emission and photon losses in the dielectric structures by way of the universal parameter of cooperativity, and thereby estimate the number of coherent cycles achievable for quantum simulation.

Because of the wide range of physical phenomena, our Article is structured to present first an overview of the parameter space that is opened by our proposals. We then describe particular examples that illuminate our strategies and analysis techniques. Details of the physics underlying these calculations are provided in the Supplementary Information.

Hubbard physics in optical lattices with $d<\lambda_{0} / 2$

Conventional investigations make use of free-space optical traps with lattice constant $d=\lambda_{0} / 2$. By moving to two-dimensional planar PCWs as in Fig. 1a, lattices with $d<\lambda_{0} / 2$ become possible for quantum simulation with both fermonic and bosonic atoms.

'Max-Planck-Institut für Quantenoptik, Hans-Kopfermann-Str. 1., 85748 Garching, Germany. ${ }^{2}$ Norman Bridge Laboratory of Physics 12-33, California Institute of Technology, Pasadena, California 91125, USA. ${ }^{3}$ Institute for Quantum Information and Matter, California Institute of Technology, Pasadena, California 91125, USA. ${ }^{4}$ ICFO-Institut de Ciencies Fotoniques, Mediterranean Technology Park, 08860 Castelldefels (Barcelona), Spain. *e-mail: hjkimble@caltech.edu 

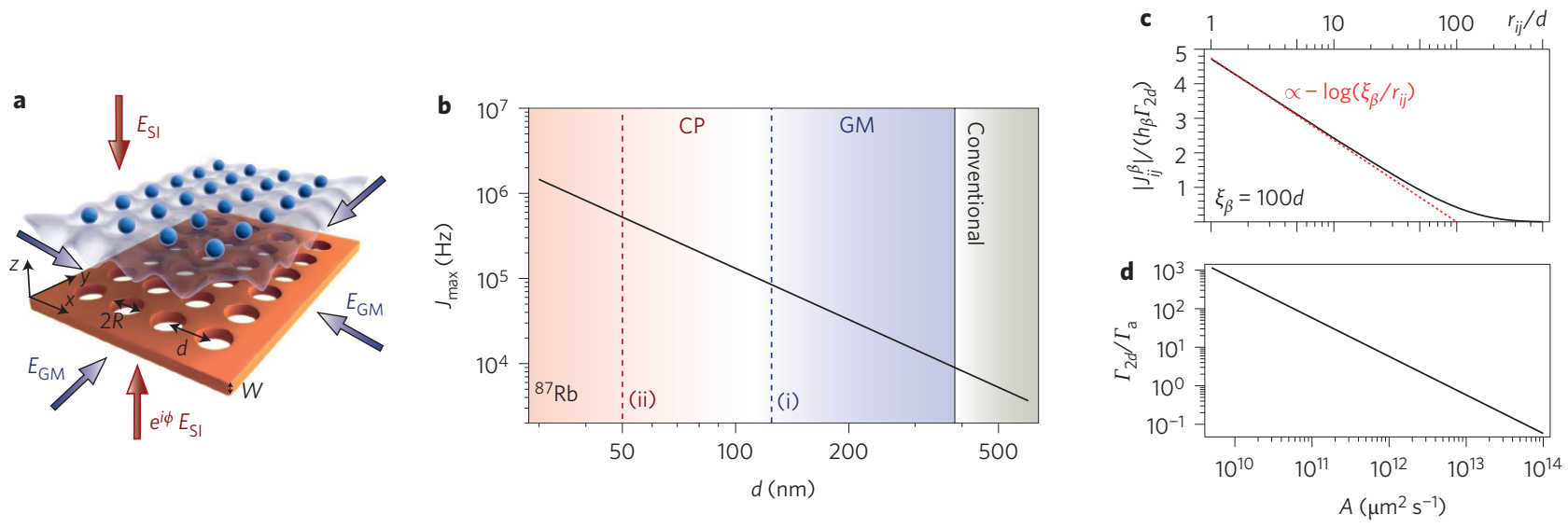

Figure 1 | Nanophotonic atom lattices and parameter regimes opened for many-body simulations. a, General scheme for a nanophotonic lattice. The dielectric slab has thickness $W$ and refractive index $n$, with a lattice of holes of radius $R$ and periodicity $d$. Optical trapping occurs by means of guided modes (GM) and side illumination (SI). b. Scaling of the maximum tunnelling ( $J$ in equation (1)) as a function of lattice periodicity $d$ for Rb. Vertical dashed lines correspond to the examples explained in the main text. c, Spatial dependence of spin-spin interaction, $\rho_{i j}^{\beta} /\left(h_{\beta} \Gamma_{2 d}\right)$, as a function of distance $r_{i j} / d$ for a situation where the atomic frequency lies in the bandgap and $\xi_{\beta}=100 \mathrm{~d}$. d, Scaling of $\Gamma_{2 d} / \Gamma_{\mathrm{a}}$ with guided-mode band curvature $A$ using the geometrical parameters of the TE mode of Figs 3 and 4 within the isotropic approximation.

For definiteness, we consider bosonic atoms, for which the following Bose-Hubbard Hamiltonian has been well established $(\hbar=1)^{26}$ :

$$
H_{\mathrm{BH}}=-J \sum_{\langle i, j\rangle} b_{i}^{\dagger} b_{j}+U \sum_{i} n_{i}\left(n_{i}-1\right) / 2
$$

where $b_{i}\left(b_{i}^{\dagger}\right)$ annihilates (creates) a localized atom on site $\mathbf{r}_{i}$, and $n_{i}=b_{i}^{\dagger} b_{i}$. The tunnelling rate $J$ and on-site interaction energy $U$ are both upper bounded by the so-called 'recoil' energy of the lattice, $E_{\mathrm{R}}=h^{2} / 8 m d^{2}$.

Figure $1 \mathrm{~b}$ presents results for the scaling of the maximum tunnelling rate $J_{\max }$ for ${ }^{87} \mathrm{Rb}$ atoms trapped in lattices with $50 \mathrm{~nm} \lesssim d \lesssim$ $300 \mathrm{~nm}$ as compared to $\lambda_{0} / 2 \approx 385 \mathrm{~nm}$ for a free-space $\mathrm{Rb}$ lattice. With only optical confinement in the GM region of Fig. 1b, the upper limit for $J, U$ could be increased by a factor of about 10 relative to a free-space lattice. Further reductions in $d$ are enabled by a novel trap design that uses vacuum forces (CP) for lateral confinement ${ }^{27,28}$ in the $x-y$ plane and optical forces along $z$ perpendicular to a planar PCW, leading to the possibility for a 60 -fold increase in $J, U$ for ${ }^{87} \mathrm{Rb}$.

\section{Strong spin-spin interactions mediated by photons}

Another exciting perspective for quantum simulation is the realization of spin-spin Hamiltonians for quantum magnetism of the general form

$$
H_{\text {spin }}=\sum_{\beta=x, y, z} \sum_{i, j} J_{i j}^{\beta} \sigma_{i}^{\beta} \sigma_{j}^{\beta}
$$

where $\sigma_{i}^{\beta}$ are Pauli operators and $J_{i j}^{\beta}$ are the spin-spin interaction energies in the $\beta$ direction for sites $i, j$. In free-space lattices, $\left|J_{i j}^{\beta}\right| / 2 \pi \lesssim 10^{3} \mathrm{~Hz}$ (ref. 29) and the range of interactions is restricted to nearest neighbours. To extend the interaction range, dipolar molecules $^{3}$ and Rydberg atoms $s^{4,5}$ yield a spatial decay of $1 / r_{i j}^{3}$. By using GMs for photon-mediated atomic interactions, we demonstrate schemes to simulate spin-models as in equation (2) with atoms trapped within two-dimensional PCWs. The dynamics can be made conservative or dissipative by varying the effective detuning $\Delta_{\beta}$ between the laser and band-edge frequencies. For the conservative regime with the atomic transition within a bandgap and $\Delta_{\beta}<0$,

$$
J_{i j}^{\beta}=h_{\beta} \Gamma_{2 d} K_{0}\left(\left|r_{i j}\right| / \xi_{\beta}\right)
$$

where $K_{0}\left(\left|r_{i j}\right| / \xi_{\beta}\right)$ is a modified Bessel function of the second kind that dictates the spatial dependence of $J_{i j}^{\beta}$. This function decays as $K_{0}(x) \propto \log (1 / x)$ when $x \ll 1$ and as $K_{0}(x) \propto e^{-x} / \sqrt{x}$ when $x \gg 1$, where $\xi_{\beta}=\sqrt{\left|A / \Delta_{\beta}\right|}$ is a tunable length (Fig. 1c) that depends both on the curvature of the bands $A$ and effective detuning $\Delta_{\beta}$. Real-time variation of $\Delta_{\beta}$ can be used to switch between the different scaling regimes. The interaction strength is $h_{\beta} \Gamma_{2 d}$, where $\Gamma_{2 d}$ describes the atom-GM coupling (Fig. 1d) and $h_{\beta}$ is fixed by the Rabi frequency and detuning of an external laser in our two-photon Raman coupling scheme (see below).

As shown in Fig. $1 \mathrm{~d}, \Gamma_{2 d} / 2 \pi \approx 10^{6}-10^{9} \mathrm{~Hz}$ (using $\Gamma_{\mathrm{a}} / 2 \pi=6.07 \mathrm{MHz}$ for the $D_{2}$ line of $\mathrm{Rb}$ in free space), depending on the geometrical and material characteristics of the PCWs (for example, curvature $A$ of the band). The projected values for $J_{i j}^{\beta}$ are several orders of magnitude larger than found with state-of-the-art methods in free-space lattices. The stronger and longer range of interactions favours frustrations and foresees the observation of more stable supersolid phases ${ }^{30-32}$, longlived metastable states ${ }^{33}$ or the 'instantaneous' transmission of correlations after local quenches ${ }^{34}$.

By contrast, operating outside the bandgap in a dispersive regime leads to dissipative evolution described by a master equation. In this case, our proposal leads to simulations of strongly long-range dissipative interactions and thereby opens new opportunities for the dissipative generation of entanglement $t^{7,35,36}$ and steady-state topological phases ${ }^{24,25}$ for two-dimensional atom lattices.

\section{Optical lattices with planar PCWs}

The regime labelled 'GMs' in Fig. $1 \mathrm{~b}$ can be studied by considering a dielectric slab with a square lattice of circular holes of periodicity $d$ as in Fig. 1a. The evanescent fields of counter-propagating GMs along $x$ and $y$ create a periodic far-off resonance optical trapping (FORT) potential proportional to $\left|\Omega_{\mathrm{GM}}(\mathbf{r})\right|^{2} / \delta$, where $\delta$ is the detuning between the GMs and atomic frequencies and $\left|\Omega_{\mathrm{GM}}(\mathbf{r})\right|^{2}$ is proportional to the field density $\left|\mathbf{E}_{\mathrm{GM}}(\mathbf{r})\right|^{2}$. As a definite example, we analyse the lowest-order transverse magnetic (TM) GMs with polarizations predominantly along $z$ for $k_{x}, k_{y}$. To stabilize the lattice in the vertical direction, a third pair of lasers counter-propagates along $z$ (side illumination, SI) with wavelength $\lambda_{\mathrm{SI}}$ and Rabi frequency $\Omega_{\mathrm{SI}}$. These $z$-beams have the same frequency but amplitudes related by $e^{i \phi}$, thereby localizing a minimum near the surface of the $\mathrm{PCW}^{17}$. The vertical trapping minima, $z_{\mathrm{t}}(x, y)$, must be near the surface of the PCW to form an $\{x, y\}$ lattice in the rapidly decaying fields of the GMs (for example, $z_{\mathrm{t}}=65 \mathrm{~nm}$ for the dashed line (i) in Fig. $1 \mathrm{~b}$, corresponding to a situation where $d=125 \mathrm{~nm}$ ). In such 

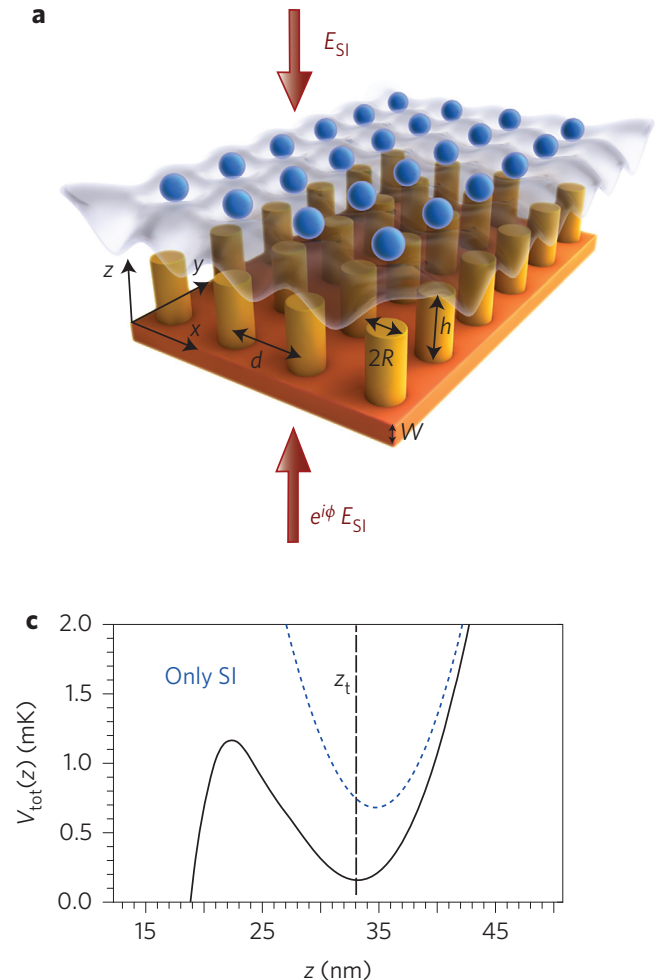

b

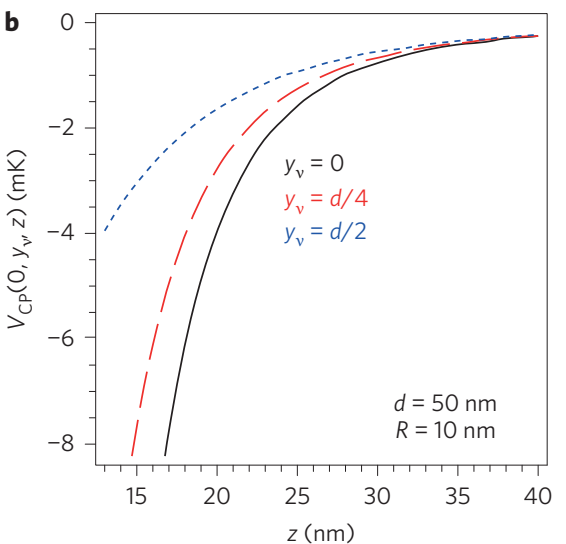

d

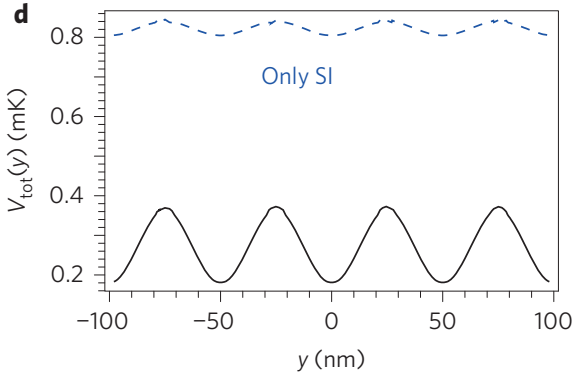

Figure 2 | Subwavelength atomic lattices using vacuum forces. a, Schematic of a dielectric slab of thickness $W$ and refractive index $n_{\text {subs }}$ with dielectric posts of height $h$ and refractive index $n_{2}$. For illustration we use GaP for the post and substrate structure and choose ${ }^{87} \mathrm{Rb}$ atoms. $\mathbf{b}, \mathrm{CP}$ potential $V_{\mathrm{CP}}(0, y, z)$ vertical cut for three different horizontal positions in the unit cell ( $y_{v}=0$ (solid black), $y_{v}=d / 4$ (dashed red) and $y_{v}=d / 2$ (dotted blue)) for a structure with $d=50 \mathrm{~nm}$ and $R=10 \mathrm{~nm}$. c, Vertical cut of the total (solid black) and SI (dotted blue) potential above the post's surface. Chirping the SI phase $\phi$ moves the $z_{\mathrm{t}}$ minimum and lattice depth. d, Horizontal cut of the total (solid black) and SI (dotted blue) potential at $z_{\mathrm{t}}=32.5 \mathrm{~nm}$. Both $\mathbf{c}$ and $\mathbf{d}$ describe $\mathrm{GaP}$ with $d=50 \mathrm{~nm}, R=0.2 d$ and $W=h=118.75 \mathrm{~nm}$. Vertical trapping is provided by SI with $\lambda_{\mathrm{SI}}=760 \mathrm{~nm}, \Omega_{\mathrm{S} I} / 2 \pi=130 \mathrm{GHz}$ for $E_{\mathrm{SI}}$ and $\phi=1.7$ rad. Horizontal trapping is provided by CP modulation.

close proximity to the dielectric, surface forces from $\mathrm{CP}$ interactions must be taken into account. The $\mathrm{CP}$ force acting on an atom depends on the atomic polarizability and the electromagnetic Green's function at the atomic position and at all frequencies ${ }^{10}$. As it is difficult to obtain effective models for geometries of such complexity, we apply the techniques described in refs 37 and 38 to evaluate the $\mathrm{CP}$ force in a numerically exact way using finitedifference time-domain (FDTD) calculations. A detailed analysis of the trapping potentials for the example of line (i) in Fig. $1 \mathrm{~b}$ is provided in Supplementary Section II.

Optimization of the lattice potential formed from GMs, SI and CP is straightforward for periodicity $\lambda_{0} / 2 n<d<\lambda_{0} / 2$, but becomes problematic for $d \lesssim \lambda_{0} / 2 n$. First note that in the limit of a uniform slab (that is, $n(r) \rightarrow n$ ), the lower bound for the periodicity in an optical lattice with guided modes is $d_{\min }=\lambda_{0} / 2 n$, obtained for a thick slab. Hence, $d_{\min }$ is reduced by a factor of $n$ relative to $d=\lambda_{0} / 2$ for freespace lattices. Reductions in $d$ below $d_{\text {min }}$ are possible for PCW optical lattices. However, PCWs rapidly lose contrast for the GM intensity and the resulting FORT potential $V_{\mathrm{GM}}(x, y)$ as $d$ decreases below $d_{\text {min }}$. Moreover, decreasing $d$ brings a requirement for trapping at $z_{\mathrm{t}}$ closer to the surface and a concomitant increase in CP forces. These two factors result in a steep rise in the laser intensities required for stable trapping (Supplementary Section II).

\section{Two-dimensional lattices with vacuum forces}

To create lattices with $d<d_{\mathrm{min}}$, we introduce a new method for trapping that uses $\mathrm{CP}$ interactions as a tool rather than a hindrance for localization near a surface. In the spirit of ref. 38, we exploit that a periodic modulation of refractive index $n(x, y)$ creates a periodic $x, y$ modulation of the $\mathrm{CP}$ potential $V_{\mathrm{CP}}(x, y, z)^{27,28}$. Large transverse wave vectors $k_{x}, k_{y}$ associated with $V_{\mathrm{CP}}\left(x, y, z_{\mathrm{t}}\right)$ for $k_{0} z_{\mathrm{t}} \ll 1$ avoid fundamental constraints on contrast for $V_{\mathrm{GM}}\left(x, y, z_{\mathrm{t}}\right)$ in subwavelength structures.

A proof-of-principle example of our scheme is shown schematically in Fig. 2a and consists of a periodic array of cylindrical posts in a deeply subwavelength regime with $d=50 \mathrm{~nm}$. The lattice for trapping in the $x-y$ plane is predominantly due to the CP potential $V_{\mathrm{CP}}(x, y, z)$, while trapping along $z$ is via $V_{\mathrm{SI}}(x, y, z)$. We compute $V_{\mathrm{CP}}(x, y, z)$ numerically ${ }^{10,37,38}$ and in Fig. $2 \mathrm{~b}$ display vertical cuts of $V_{\mathrm{CP}}\left(0, y_{\mathrm{v}}, z\right)$ at $y_{\mathrm{v}}=\{0, d / 2, d\}$, showing the $z$-dependence of $V_{\mathrm{CP}}\left(0, y_{\mathrm{v}}, z\right)$ as the planar position moves from the axis of a rod at $\{x, y\}=0$. Although we focus on a unit cell, a two-dimensional array of posts is included in our calculation. The total trapping potential for the atomic lattice is $V_{\text {tot }}(x, y, z)=V_{\mathrm{CP}}(x, y, z)+V_{\mathrm{SI}}(x, y, z)$, with line cuts shown in Fig. 2 c,d. For Fig. $2 c$, the trap minima are chosen to be at a vertical distance of $z_{\mathrm{t}} \approx 32.5 \mathrm{~nm}$ to achieve the required contrast in the $x-y$ plane for the Bose-Hubbard physics of equation (1). Figure $2 \mathrm{~d}$ clearly demonstrates that the dominant contribution to the variation of $V_{\text {tot }}\left(x, y, z_{\mathrm{t}}\right)$ in the $x-y$ plane is the 'vacuum lattice' from CP interactions and not $V_{\mathrm{SI}}\left(x, y, z_{\mathrm{t}}\right)$.

The trap depth of the two-dimensional vacuum lattice can be dynamically tuned over a wide range by adjusting the vertical trap position $z_{\mathrm{t}}$. The trap depth $V_{\mathrm{d}}$ and frequencies $\omega_{\mathrm{t}}$ for Fig. 2 are $\left\{V_{\mathrm{d}, x y}, V_{\mathrm{d}, \mathrm{z}}\right\} / 2 \pi \approx\{3.5,20.8\} \mathrm{MHz}$ and $\left\{\omega_{\mathrm{t}, x y}, \omega_{\mathrm{t}, \mathrm{z}}\right\} / 2 \pi \approx\{1.7,4.2\} \mathrm{MHz}$. In the $x-y$ plane, the trapping depth of Fig. 2 is $\sim 15 E_{\mathrm{R}}$, which guarantees the possibility of having localized Wannier modes in the lattice $^{39}$. If we used only the SI FORT potential, the trap depth would be $\sim 3 E_{\mathrm{R}}$, which does not lead to localization in a unit cell. 

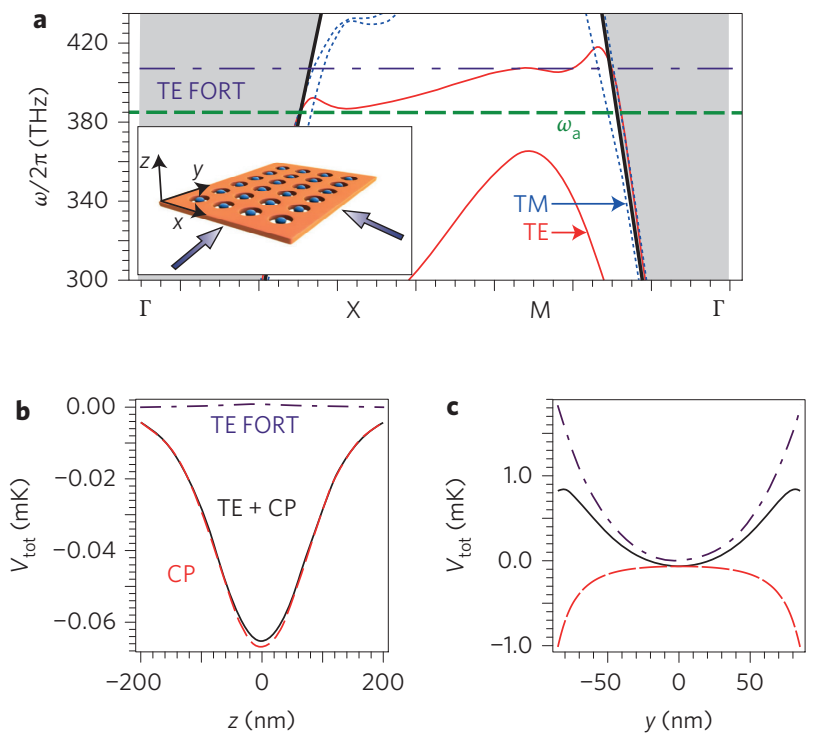

Figure 3 | Scheme for atom trapping to enable photon-mediated atomic interactions. a, Band structure of a GaP photonic crystal slab with a square lattice of holes with $W=0.316 d, R=0.4 d$ and $d=316 \mathrm{~nm}$. Dashed green line: atomic frequency. Dashed-dotted purple line: GM frequency for trapping. Thick black line: the light line above which the modes are non-guided. Inset: general scheme for atom trapping inside holes of the structure. $\mathbf{b}$, Total potential (black) and its different contributions: TE FORT (dashed-dotted purple) and CP (dashed red) as a function of vertical distance $z$, with origin $z=0$ in the centre of the hole. $\mathbf{c}$, As in $\mathbf{b}$, but for a horizontal cut in $y$ at the centre of the hole $z=x=0 . \lambda_{0}$ and $\Omega_{\mathrm{GM}} / 2 \pi$ are $\sim 730 \mathrm{~nm}$ and $35 \mathrm{GHz}$, respectively.

The associated scattering rate of the trap scales as $V_{\mathrm{SI}} \Gamma_{\mathrm{a}} / \delta$, yielding scattering rates of $\sim 2 \pi \times 10 \mathrm{~Hz}$ for $\mathrm{Rb}$. The results in Fig. 2, corresponding to Fig. 1b,ii, and further analyses (Supplementary Section III) suggest that vacuum lattices could provide significant increases in the energy scale for Bose-Hubbard physics with cold atoms.

\section{Long-range interactions mediated by photons in PCWs}

Our discussion thus far relates to the CP and GM regimes in Fig. 1b, with an emphasis on a reduced lattice constant relative to free space. However, PCWs also offer exciting opportunities for investigations of many-body physics via photon-mediated interactions among atoms trapped within a PCW ${ }^{11}$. Although one-dimensional PCWs have predominantly been considered ${ }^{14,18,19,22,38,40}$, our schemes for two-dimensional PCWs yield physics beyond the one-dimensional case. Moreover, we provide full descriptions of the trapping configuration, band design and photon-mediated couplings using realistic parameters.

Figure 3a presents the band structure for a square lattice of holes in a GaP slab ${ }^{41}$. Our interest is in the GMs shown in the area below the light line, while the shaded area above the light line represents the continuum of leaky modes. This structure supports a bandgap for the transverse electric (TE-like) GMs (in red). To achieve photonmediated atomic interactions, we design the band structure to provide a band of GMs suitable for atom trapping and for large atom-field interactions. Off-resonant excitation with TE-guided modes along $\left\{k_{x}, k_{y}\right\}$ near the M-point of the band diagram creates a FORT lattice in the $x-y$ plane that compensates the CP forces in these directions. Atoms are trapped at $z_{\mathrm{t}}=0$ using vertical confinement provided by CP forces ${ }^{38}$. By further including two SI beams counter-propagating along $z$, it is also possible to control the position of the minima in $z$ and load the trap. Line cuts of the total trap potential $V_{\text {tot }}$ are given in Fig. 3b,c, with the contributions from the
FORT (purple) and CP (red) potentials shown. The trap depth $V_{\mathrm{d}}$ and frequencies $\omega_{\mathrm{t}}$ for the particular example in Fig. 3 are $\left\{V_{\mathrm{d}, x y}\right.$, $\left.V_{\mathrm{d}, \mathrm{z}}\right\} / 2 \pi \approx\{18.7,1.3\} \mathrm{MHz}$ and $\left\{\omega_{\mathrm{t}, x y}, \omega_{\mathrm{t}, \mathrm{z}}\right\} / 2 \pi \approx\{0.67,0.16\} \mathrm{MHz}$.

The $\mathrm{Rb}$ atoms trapped in the centres of the holes in Fig. $3 \mathrm{~b}, \mathrm{c}$ interact with TE guided modes near the band edge at the $\mathrm{X}$ point, with guided mode frequencies approaching that of the $D_{2}$ line of $\mathrm{Rb}$. As shown in Fig. 4a we consider an atomic $\Lambda$ scheme driven by two off-resonant lasers with detunings $\Delta_{l}=\left(\omega_{e}-\omega_{g_{l}}\right)-\omega_{L, l}$ for $l=1,2$. The polarizations are chosen such that the transition $g_{1} \leftrightarrow e$ interacts with the TE guided modes near the $\mathrm{X}$ point (which are polarized predominantly along $y, x$ for $k_{x}, k_{y}$ ). $g_{2} \leftrightarrow e$ interacts instead with TM-like modes (which are polarized principally along the $z$-direction). Mode profile intensities are shown in Fig. 4c,d, with large (small) coupling strength at the trap site for the TE (TM) guided modes with energies close to $\omega_{\mathrm{a}}=\omega_{e}-\omega_{g_{1}}$, which lies within the bandgap.

Assuming that the coupling with the guided modes can be treated perturbatively and in the limit where $\left|\Delta_{l}\right| \gg \Omega_{l}(l=1,2)$ for the scheme of Fig. $4 \mathrm{a}$, both the excited states and the guided modes can be adiabatically eliminated, leading to interactions betweeen the two-state spins $\left\{\left|g_{1}\right\rangle_{i},\left|g_{2}\right\rangle_{i}\right\}$, which results in an effective master equation ${ }^{42}$ :

$$
\dot{\rho}=\sum_{i, j} \sum_{\beta=x y, z}\left[\Gamma_{i j}^{\beta}\left(\mathcal{O}_{i}^{\beta} \rho\left(\mathcal{O}_{j}^{\beta}\right)^{\dagger}-\left(\mathcal{O}_{j}^{\beta}\right)^{\dagger} \mathcal{O}_{i}^{\beta} \rho\right)+\text { h.c }\right]
$$

where $\Gamma_{i j}^{\beta}=\gamma_{i j}^{\beta} / 2+i J_{i j}^{\beta}=h_{\beta} \Gamma_{2 d} F^{\beta}\left(r_{i j}\right)$ and $\mathcal{O}_{i}^{\beta}=\left|g_{1}\right\rangle_{i}\left\langle g_{2}\right|, \sigma_{i}^{z}$ for $\beta=x y, z$, respectively. Here $h_{\beta}=\left(\Omega_{l} /\left(2 \Delta_{\beta}\right)\right)^{2}$, with $l=1(2)$ for $\beta=z(x y) . F^{\beta}\left(r_{i j}\right)$ is a function whose form depends on whether $\left\{\Delta_{x y}, \Delta_{z}\right\} \lessgtr 0$, where $\Delta_{x y}=\omega_{g, 2}-\omega_{g, 1}+\omega_{L, 2}-\omega_{c}$ and $\Delta_{z}=\omega_{L, 1}-\omega_{c}$ (Supplementary Section IV). The imaginary contribution of the collective coupling, $J_{i j}^{\beta}$, accounts for the coherent evolution, and the real part, $\gamma_{i j}^{\beta}$, describes collective dissipation.

To find approximate expressions for these spin-spin interactions we use a parabolic approximation of $\omega(\mathbf{k})$ as depicted in Fig. $4 \mathrm{~b}$ and assume that around the X point both the coupling and $\omega(\mathbf{k})$ behave isotropically. We have also performed numerical integration with the exact energy dispersion and coupling of the structure obtaining similar scalings (Supplementary Section IV). When $\Delta_{\beta}>0, F^{\beta}\left(r_{i j}\right)$ has both real and imaginary components given by

$$
\left.\Gamma_{i j}^{\beta}\right|_{\Delta_{\beta}>0}=\frac{\pi}{2} h_{\beta} \Gamma_{2 d} H_{0}^{(1)}\left[\left|r_{i j}\right| / \xi_{\beta}\right]
$$

where $H_{0}^{(1)}(x)=J_{0}(x)+i Y_{0}(x)$ is a Hankel function of the first kind and the length scale $\xi_{\beta}=\sqrt{\left|A / \Delta_{\beta}\right|}$ determines the range and strength of the correlations that can be controlled independently via the detuning $\Delta_{\beta}$ (ref. 22). The Bessel function $J_{0}(x) \propto 1$ for $x \ll 1$ and $\propto 1 / \sqrt{x}$ for $x \gg 1$.

By contrast, when $\Delta_{\beta}<0, \Gamma_{i j}^{\beta}$ are purely imaginary: $\left.\Gamma_{i j}^{\beta}\right|_{\Delta_{\beta}<0}=i j_{i j}^{\beta}$, with $J_{i j}^{\beta}$ as defined in equation (3). Ultimately, the modified Bessel function $K_{0}(x)$ is damped by an exponential factor controlled by $\xi_{\beta}$, which can be tuned dynamically through the detuning $\Delta_{\beta}$ and made large enough to guarantee that we reach the limit $x=r_{i j} / \xi_{\beta} \ll 1$, where $J_{i j}^{\beta}$ is of strongly long-range character, as depicted in Fig. 1c. In this regime, we then engineer the following general class of XXZ spin Hamiltonians:

$$
H=\sum_{i, j}\left[J_{i j}^{z} \sigma_{i}^{z} \sigma_{j}^{z}+J_{i j}^{x y} \sigma_{i}^{\dagger} \sigma_{j}\right]
$$

where $J_{i j}^{z(x y)}$ can be tuned independently by changing the laser intensities, $\Omega_{1}$ or effective detunings $\Delta_{\beta}$.

Although conceptually straightforward to place an atomic transition within a bandgap for interactions mediated by virtual photons $s^{11,14,22}$, this is problematic in practice due to the difficulty 

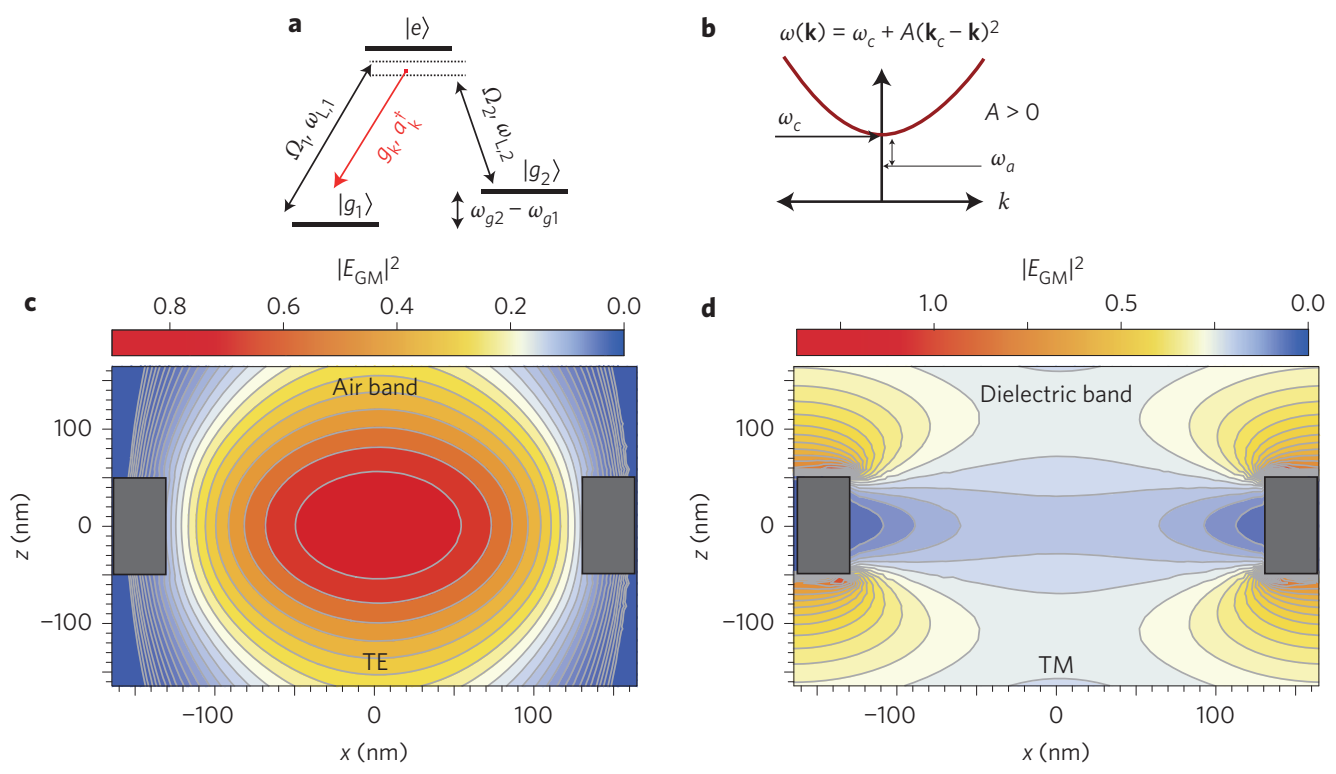

Figure 4 | Atomic level structure, simplified dispersion relation and GM intensities. a, Lambda configuration to engineer the coupling to a single polarization of the PCW continuum. b, Parabolic approximation of the band structure close to a band edge. $\mathbf{c}, \mathbf{d},\left|E_{\mathrm{GM}}(x, 0, z)\right|^{2}$ for the TE (c) and TM (d) polarized guided modes of the air (c) and dielectric (d) bands with frequency around $\omega_{\mathrm{a}}$ of a GaP PCW slab with a square lattice of holes with $d=316 \mathrm{~nm}$, $R=0.4 d$ and $W=0.316 d$. Dark shaded areas represent the regions of dielectric material.

of obtaining overlapping bandgaps for TE and TM modes in lowloss dielectrics in the optical domain. Our configuration relies on the existence of a bandgap for only a single polarization of the structure. It also allows for dynamical tuning of the interaction parameters near the band edge $\mathrm{e}^{22}$.

The strength of atom-atom interactions in the PCW is ultimately determined by $\Gamma_{2 d}$ and a logarithmic correction coming from $K_{0}$ that scales approximately as $\log \left(d / \xi_{\beta}\right)$ and contains the dependence with $\Delta_{\beta}$. For the regime $\Delta_{\beta}<0$ (that is, to obtain purely coherent spinspin interactions), we find that (Supplementary Section IV)

$$
\Gamma_{2 d} \approx \Gamma_{\mathrm{a}} \frac{c \sigma}{4 \pi A L_{m}\left(\omega_{c}, r_{\mathrm{a}}\right)}
$$

where $\Gamma_{\mathrm{a}}$ is the free-space radiative decay rate, $\sigma=(3 / 2 \pi) \eta \lambda_{\mathrm{a}}^{2}$ is the effective cross-section, $\eta$ is a correction parameter that depends on the atomic implementation and $L_{m}\left(\omega, \mathbf{r}_{\mathrm{a}}\right)$ is the effective mode length, which depends on both the atomic position and the electric field density of the guided mode. In Fig. $1 \mathrm{~d}$ we plot the scaling of $\Gamma_{2 d}$ against the curvature parameter $A$, using the effective length of the structure in Figs 3 and 4 in the hole centre, $L_{\mathrm{m}, \mathrm{TE}} \approx 0.3 \mu \mathrm{m}$ and $\eta=1 / 2$. The averaged band curvature of our structure in Fig. 3 is $A \approx 1.8 \times 10^{12} \mu \mathrm{m}^{2} \mathrm{~s}^{-1}$. However, to obtain more accurate estimates of $J_{i j}^{\beta}$ we performed numerical calculations taking into account the actual anisotropic band structure, as well as details of the atomic implementation, thereby obtaining $J_{i j}^{\beta}$ of the order $30-40 \Gamma_{\mathrm{a}}$ for detunings such that $\xi_{\beta} \approx 100 d$ (Supplementary Section IV).

Beyond the processes described by equations (4) to (7), there will be a variety of mechanisms that lead to decoherence for photonmediated atomic interactions. Without a full three-dimensional bandgap for our two-dimensional structures, trapped atoms will radiatively decay into free-space and lossy modes within the PCW. To estimate these losses, we performed FDTD simulations for structures as in Figs 3 and 4 and found that the total atomic decay rate to all channels except the designated guided modes is $\Gamma^{\prime} \approx 0.4 \Gamma_{\mathrm{a}}$. The PCW itself will have imperfections that can be estimated from the observed quality factors $Q$ for state-of-the-art nanophotonic structures ${ }^{43-45}$, which recently reported a $Q$ of $\sim 10^{6}-10^{7}$.
This finite $Q$ translates into a finite photon lifetime, $\kappa=\omega_{c} / Q$, which together with $\Gamma^{\prime}$, leads to an effective rate of decoherence $\kappa_{\text {eff }}=\Gamma^{\prime}+$ $\kappa J_{i j} / \Delta_{\beta}$ (ref. 22). Intuitively, the number of spin-exchange cycles in the presence of decoherence can be characterized by $N=J_{i j} / \kappa_{\text {eff }}$. Using our structure as in Figs 3 and 4 and taking $Q=10^{7}, N \approx 35$ is obtained for a detuning of $\Delta_{\beta} \approx 10 \mathrm{GHz}$, which yields $J_{i j} \approx 16 \Gamma_{\mathrm{a}}$ and $\xi_{\beta} \approx 16 d$ (Supplementary Section IV). Further improvements in the material quality and alternative lattice geometries could provide flatter bands (that is, reduced $A$ ) and better $Q$, thereby increasing both $N$ and the effective interaction strength and length.

\section{Conclusion}

We have shown how two-dimensional PCWs can be used to trap atoms and realize new kinds of subwavelength optical lattices with higher tunnelling rates for simulations of Bose-Hubbard physics. Moreover, the possibility of combining atom trapping with photon-mediated atom-atom interactions via two-dimensional guided modes in PCWs enables realizations of spin models with large, tunable and strong long-range interactions of both dissipative and coherent character. Beyond the particular examples in Figs 1-4, we have developed designs for a variety of other structures (for example, dielectric posts instead of holes and triangular instead of square lattices) and materials (for example, $\mathrm{TiO}_{2}, \mathrm{SiC}$ and $\mathrm{SiN}$ ) with comparable performance to those described here. Our extensive investigations with diverse structures and materials support the applicability of our projections in Fig. $1 \mathrm{~b}-\mathrm{d}$ to a wide class of problems as in equations (1) and (2).

Received 15 June 2014; accepted 2 March 2015; published online 6 April 2015

\section{References}

1. Bloch, I., Dalibard, J. \& Nascimbène, S. Quantum simulations with ultracold quantum gases. Nature Phys. 8, 267-276 (2012).

2. Cirac, J. I. \& Zoller, P. Goals and opportunities in quantum simulation. Nature Phys. 8, 264-266 (2012).

3. Micheli, A., Brennen, G. \& Zoller, P. A toolbox for lattice-spin models with polar molecules. Nature Phys. 2, 341-347 (2006).

4. Jaksch, D. et al. Fast quantum gates for neutral atoms. Phys. Rev. Lett. 85, 2208-2211 (2000) 
5. Lukin, M. D. \& Ǐmamoglu, A. Controlling photons using electromagnetically induced transparency. Nature 413, 273-276 (2001).

6. Bouchet, F., Gupta, S. \& Mukamel, D. Thermodynamics and dynamics of systems with long-range interactions. Physica A 389, 4389-4405 (2010).

7. Gullans, M. et al. Nanoplasmonic lattices for ultracold atoms. Phys. Rev. Lett. 109, 235309 (2012).

8. Romero-Isart, O., Navau, C., Sanchez, A., Zoller, P. \& Cirac, J. I. Superconducting vortex lattices for ultracold atoms. Phys. Rev. Lett. 111, 145304 (2013).

9. Joannopoulos, J. D., Johnson, S. G., Winn, J. N. \& Meade, R. D. Photonic Crystals: Molding the Flow of Light (Princeton Univ. Press, 2011).

10. Buhmann, S. Y. \& Welsch, D.-G. Dispersion forces in macroscopic quantum electrodynamics. Prog. Quant. Electron. 31, 51-130 (2007).

11. John, S. \& Wang, J. Quantum electrodynamics near a photonic band gap: photon bound states and dressed atoms. Phys. Rev. Lett. 64, 2418-2421 (1990).

12. Kurizki, G. Two-atom resonant radiative coupling in photonic band structures. Phys. Rev. A 42, 2915-2924 (1990).

13. Lambropoulos, P., Nikolopoulos, G. M., Nielsen, T. R. \& Bay, S. Fundamental quantum optics in structured reservoirs. Rep. Prog. Phys. 63, 455-503 (2000).

14. Shahmoon, E. \& Kurizki, G. Nonradiative interaction and entanglement between distant atoms. Phys. Rev. A 87, 033831 (2013).

15. Vetsch, E. et al. Optical interface created by laser-cooled atoms trapped in the evanescent field surrounding an optical nanofiber. Phys. Rev. Lett. 104, 203603 (2010).

16. Goban, A. et al. Demonstration of a state-insensitive, compensated nanofiber trap. Phys. Rev. Lett. 109, 033603 (2012).

17. Thompson, J. D. et al. Coupling a single trapped atom to a nanoscale optical cavity. Science 340, 1202-1205 (2013).

18. Goban, A. et al. Atom-light interactions in photonic crystals. Nature Commun. 5, 3808 (2014).

19. Yu, S.-P. et al. Nanowire photonic crystal waveguides for single-atom trapping and strong light-matter interactions. Appl. Phys. Lett. 104, 111103 (2014)

20. Tiecke, T. et al. Nanophotonic quantum phase switch with a single atom. Nature 508, 241-244 (2014).

21. Chang, D. E., Cirac, J. I. \& Kimble, H. J. Self-organization of atoms along a nanophotonic waveguide. Phys. Rev. Lett. 110, 113606 (2013)

22. Douglas, J. S. et al. Quantum many-body models with cold atoms coupled to photonic crystals. Nature Photon. http://dx.doi.org/10.1038/nphoton.2015.57 (2015).

23. Cho, J., Angelakis, D. G. \& Bose, S. Fractional quantum Hall state in coupled cavities. Phys. Rev. Lett. 101, 246809 (2008).

24. Diehl, S., Rico, E., Baranov, M. A. \& Zoller, P. Topology by dissipation in atomic quantum wires. Nature Phys. 7, 971-977 (2011).

25. Bardyn, C. et al. Topology by dissipation. New J. Phys. 15, 085001 (2013)

26. Jaksch, D., Bruder, C., Cirac, J. I., Gardiner, C. W. \& Zoller, P. Cold bosonic atoms in optical lattices. Phys. Rev. Lett. 81, 3108-3111 (1998).

27. Yannopapas, V. Optical forces near a plasmonic nanostructure. Phys. Rev. B 78, 045412 (2008).

28. Contreras-Reyes, A. M. et al. Casimir-Polder interaction between an atom and a dielectric grating. Phys. Rev. A 82, 052517 (2010).

29. Trotzky, S. et al. Time-resolved observation and control of superexchange interactions with ultracold atoms in optical lattices. Science 319, 295-299 (2008).

30. Büchler, H. P. et al. Strongly correlated $2 \mathrm{D}$ quantum phases with cold polar molecules: controlling the shape of the interaction potential. Phys. Rev. Lett. 98, 060404 (2007).

31. Hauke, P. et al. Complete devil's staircase and crystal-superfluid transitions in a dipolar XXZ spin chain: a trapped ion quantum simulation. New J. Phys. 12, 113037 (2010).

32. Maik, M., Hauke, P., Dutta, O., Zakrzewski, J. \& Lewenstein, M. Quantum spin models with long-range interactions and tunnelings: a quantum Monte Carlo study. New J. Phys. 14, 113006 (2012).
33. Trefzger, C., Menotti, C. \& Lewenstein, M. Ultracold dipolar gas in an optical lattice: the fate of metastable states. Phys. Rev. A 78, 043604 (2008).

34. Hauke, P. \& Tagliacozzo, L. Spread of correlations in long-range interacting quantum systems. Phys. Rev. Lett. 111, 207202 (2013).

35. Verstraete, F., Wolf, M. M. \& Cirac, J. I. Quantum computation and quantumstate engineering driven by dissipation. Nature Phys. 5, 633-636 (2009).

36. González-Tudela, A. \& Porras, D. Mesoscopic entanglement induced by spontaneous emission in solid-state quantum optics. Phys. Rev. Lett. 110, 080502 (2013).

37. Rodriguez, A. W., McCauley, A. P., Joannopoulos, J. D. \& Johnson, S. G. Casimir forces in the time domain: theory. Phys. Rev. A 80, 012115 (2009).

38. Hung, C.-L., Meenehan, S. M., Chang, D. E., Painter, O. \& Kimble, H. J. Trapped atoms in one-dimensional photonic crystals. New J. Phys. 15, 083026 (2013).

39. Greiner, M. Ultracold Quantum Gases in Three-Dimensional Optical Lattice Potentials PhD thesis, Ludwig-Maximilians-Universität München (2003).

40. Shen, J.-T. \& Fan, S. Coherent single photon transport in a one-dimensional waveguide coupled with superconducting quantum bits. Phys. Rev. Lett. 95, 213001 (2005).

41. Johnson, S. G. \& Joannopoulos, J. D. Block-iterative frequency-domain methods for Maxwell's equations in a planewave basis. Opt. Express 8, 173-190 (2001).

42. Breuer, H.-P. \& Petruccione, F. The Theory of Open Quantum Systems (Oxford Univ. Press, 2002).

43. Srinivasan, K. \& Painter, O. Momentum space design of high- $Q$ photonic crystal optical cavities. Opt. Express 10, 670-684 (2002).

44. Taguchi, Y., Takahashi, Y., Sato, Y., Asano, T. \& Noda, S. Statistical studies of photonic heterostructure nanocavities with an average $Q$ factor of three million. Opt. Express 19, 11916-11921 (2011).

45. Sekoguchi, H., Takahashi, Y., Asano, T. \& Noda, S. Photonic crystal nanocavity with a Q-factor of 9 million. Opt. Express 22, 916-924 (2014).

\section{Acknowledgements}

The authors thank O. Painter, J. Muñiz and S. Meenehan for discussions. The work of A.G.-T. and J.I.C. was funded by European Union integrated project 'Simulators and Interfaces with Quantum Systems' (SIQS). A.G.-T. also acknowledges support from the Alexander Von Humboldt Foundation and Intra-European Fellowship NanoQuIS (625955). J.I.C. acknowledges support as a Moore Distinguished Scholar. D.E.C. acknowledges support from Fundacio Privada Cellex Barcelona. H.J.K. and C.-L.H. acknowledge funding by the Institute for Quantum Information and Matter, a National Science Foundation (NSF) Physics Frontier Center, with support of the Moore Foundation, by the Air Force Office of Scientific Research, Quantum Memories in Photon-Atomic-Solid State Systems (QuMPASS) Multidisciplinary University Research Initiatives (MURI), by the Department of Defense National Security Science and Engineering Faculty Fellows (DoD NSSEFF) programme, and by NSF PHY1205729. H.J.K. acknowledges support as a Max Planck Institute for Quantum Optics Distinguished Scholar.

\section{Author contributions}

A.G.-T. and C.-L.H. carried out analytical and numerical calculations. A.G.-T., C.-L.H., D.C., J.I.C. and H.J.K. contributed materials and analysis tools. A.G-T. and H.J.K. wrote the manuscript.

\section{Additional information}

Supplementary information is available in the online version of the paper. Reprints and permissions information is available online at www.nature.com/reprints. Correspondence and requests for materials should be addressed to H.J.K.

\section{Competing financial interests}

The authors declare no competing financial interests. 